\title{
Endplate Changes with Polyetheretherketone Cages in Posterior Lumbar Interbody Fusion
}

\author{
Tarek Anwar Elfiky ${ }^{1, *}$, Nirmal Dhananjay Patil ${ }^{2, *}$, Yasser Allam ${ }^{1}$, Raafat Ragab ${ }^{1}$ \\ ${ }^{1}$ Spine Unit, Al-Hadra University Hospital, Alexandria, Egypt \\ ${ }^{2}$ Department of Orthopaedics, Seth GS Medical College and KEM Hospital, Mumbai, India
}

\begin{abstract}
Study Design: A retrospective radiographic analysis.
Purpose: The aim of the current study is to assess endplate changes after the use of polyetheretherketone (PEEK) cages in posterior lumbar interbody fusion (PLIF).

Overview of Literature: A few recent reports had revealed endplate abnormalities due to PEEK cages, which may lead to nonunions.

Methods: A retrospective computed tomography (CT)-based analysis of the endplate cavities and fusion status following PLIFs with PEEK cages was conducted by two independent observers. The term "cavity" was used to describe the endplate changes. The vertebral endplate cavities were assessed according to the size, multiplicity, location, and presence or absence of sclerosis.

Results: There were 86 fixed levels in 65 consecutive patients, with a mean age of $35.44 \pm 19.60$ years. The mean follow-up was 16.5 \pm 10.1 months (range, 6-57 months). Definite fusion was seen in 56 levels (65.12\%) by observer 1 versus 44 levels (51.16) by observer 2. The strength of agreement was moderate. Endplate cavities were observed in 42 levels (48.84\%) by observer 1 versus 47 levels $(54.65 \%)$ by observer 2, with fair agreement. The strengths of agreement for the locations, multiplicity, and size were moderate, fair, and poor, respectively. Neither age, sex, etiology, levels, nor follow-up period was significantly associated with the presence of cavities. With regard to fusions, the nonunions detected by observer 1 were significantly associated with the presence of cavities $(p<0.0001)$. However, those detected by observer 2 were nearly significant $(p=0.05)$.

Conclusions: There was a high rate of unfavorable radiographic findings in the form of endplate cavities in PLIF cases with PEEK cages. A more comprehensive classification for the assessment of fusions and endplate cavities should be formulated. We strongly recommend further CT-based studies with larger sample size and longer follow-up periods.
\end{abstract}

Keywords: Interbody; Fusion; Polyetheretherketone; End plate changes; Cavities

\section{Introduction}

Posterior lumbar interbody fusion (PLIF) is a standard procedure for the treatment of different lumbar degenerative disorders. The goals of fusion procedures using cages/spacers with posterior instrumentation and grafts are to stabilize the motion segment and to facilitate the fusion process. Spacers were initially manufactured using either stainless steel or titanium alloys [1-3]. Metal cages showed good fusion rates [4,5]; however, three problems

Received Apr 26, 2019; Revised May 26, 2019; Accepted Jun 12, 2019

Corresponding author: Tarek Anwar Elfiky

Spine Unit, Orthopedic Department, Al-Hadra University Hospital, Amprozo, Alexandria, Egypt

Tel: +20-00201223382958, Fax:+20-0025932907, E-mail: tarekfiky@yahoo.com

*These two authors contributed equally to this work. 
have been associated with their use. These are subsidence of the cage into the adjacent vertebrae, difficulties in assessing fusion by imaging, and stiffness of the material that reduces the amount of mechanical stimulation to the bone grafts, which might delay the fusion or shielding of the bone graft [6-11]. To overcome these shortcomings, polyetheretherketone (PEEK) cages have been introduced. PEEK cages have several advantages over metal, including the lack of metal allergies and moderate stiffness, which has a modulus of elasticity similar to that of human cortical bone that may increase the union rate of the grafted bone by minimizing stress-shielding effects. Moreover, the radiolucency of PEEK cages results in more reliable evaluations of fusion on computed tomography (CT) images, allowing for a clearer, unobstructed view of new bone formation during the follow-up examination and low numbers of artifacts on magnetic resonance imaging $[12,13]$. However, there is insufficient evidence supporting the superiority of PEEK cages over metal cages as optimal interbody spacers $[14,15]$. Only a few articles have recently demonstrated unfavorable radiographic endplate findings with the use of PEEK cages, which may lead to nonunion $[15,16]$.

The aim of the current study is to assess the endplate changes resulting from the use of PEEK cages in PLIFs.

\section{Materials and Methods}

\section{Patients}

The study protocol was approved by the Institutional Review Board and Ethics Committee (IRB approval no., 12814). Informed consent was obtained from each patient.

A database of consecutive patients who underwent single or multi-level PLIFs with PEEK cages for different lumbar disorders at Spine Surgery Unit, Orthopedics and Traumatology, Alexandria University between January 2012 and December 2014 was retrospectively analyzed. The primary diagnoses were degenerative or isthmic spondylolisthesis, degenerative disc disease, lumbar stenosis, lumbar disc herniation, or recurrent lumbar disc herniation. We excluded patients with spinal infections, tumors, or trauma and those who lacked sufficient radiographic data. Additionally, cases with known postoperative infections were excluded from our study.

\section{Surgical considerations}

All cases were performed by two experienced senior consultant spinal surgeons. PLIFs were conducted in a standard way using PEEK cages with pedicle screw fixations. Locally, the adjacent cartilage endplates were removed as fully as possible, but the bony endplates were preserved. Only locally harvested bone obtained during the decompression procedure was grafted inside and around the cage. All cages were either bullet-shaped or kidney-shaped (Spineart Co., Geneva, Switzerland). Recombinant bone morphogenetic protein (rhBMP), other bone-stimulating agents, or bone substitutes were not used in this study. All patients were mobilized from the second or third postoperative day without a lumbar brace.

\section{Radiologic evaluation}

\section{1) Assessment of bone union}

A 64-line multi-slice CT scanner with 1-mm-thick axial helical slices was used to evaluate all patients. Bony union was assessed by multi-slice CTs 6 and 12 months after the surgery or until solid fusion was achieved.

The criteria for radiological assessment were as follows: (1) Solid fusion was defined as the presence of complete bridging bone within and around the cage in at least one cut on the coronal and sagittal CT images. (2) Questionable fusion was defined as the presence of incomplete bridging bone growing from both vertebral endplates. (3) Non-fusion was defined as the lack of bony bridges, screw loosening, or breakage. (4) Complications such as cage migration or retropulsion, screw loosening, or breakage were also recorded.

2) Classification of vertebral endplate changes (cavities) Vertebral endplate changes were previously described as osteolysis [15] or cysts [16]. Since we had no histological evidence from the literature regarding the nature of these changes, the term "cavities" was used in this study. A vertebral endplate cavity was defined as a visible lowdensity circular or oval area on the sagittal and/or coronal CT images located at the cranial and/or caudal vertebral endplate around or outside the cage. They were assessed according to the following criteria: (1) The size of the cavity area was judged as I: small $(<5 \mathrm{~mm})$, II: moderate (5-10 mm), or III: large (>10 mm). (2) The location was described as in the cephalic or caudal endplates or both. (3) 

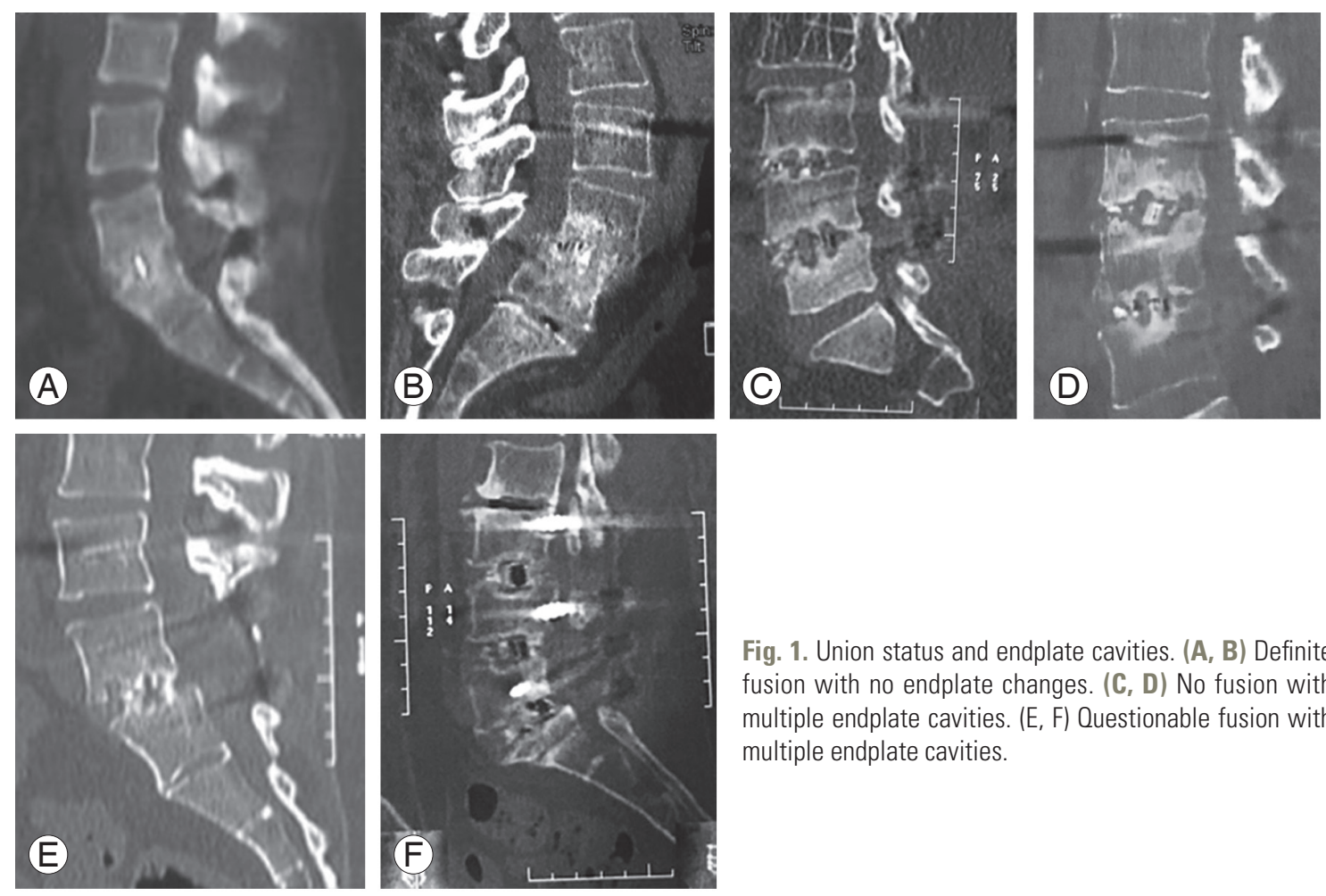

Fig. 1. Union status and endplate cavities. (A, B) Definite fusion with no endplate changes. (C, D) No fusion with multiple endplate cavities. (E, F) Questionable fusion with multiple endplate cavities.

The presence or absence of sclerosis around the cavity was noted.

All imaging studies were blindly and independently reviewed by the two senior consultant authors who had performed the surgeries.

\section{Statistical analyses}

Statistical analyses were performed using IBM SPSS software ver. 19.0 (IBM Corp., Armonk, NY, USA). The quantitative data are presented as the mean \pm standard deviation. Pearson's correlation test was performed to determine correlations between the parameters. The kappa ( $\kappa)$ coefficient between the two independent observers was calculated to determine the interobserver reliability. Significance was defined as a $p$-value less than 0.05 .

\section{Results}

A total of 65 consecutive patients (21 males and $44 \mathrm{fe}$ males) were enrolled in the study. The average age at surgery was $35.44 \pm 19.60$ years (range, 26-67 years). The number of levels fixed was 86 . The most frequently fixed levels were L4-L5 in 38 levels (44.19\%) and L5-S1 in 36 levels $(41.86 \%)$. The mean follow-up was $16.5 \pm 10.1$

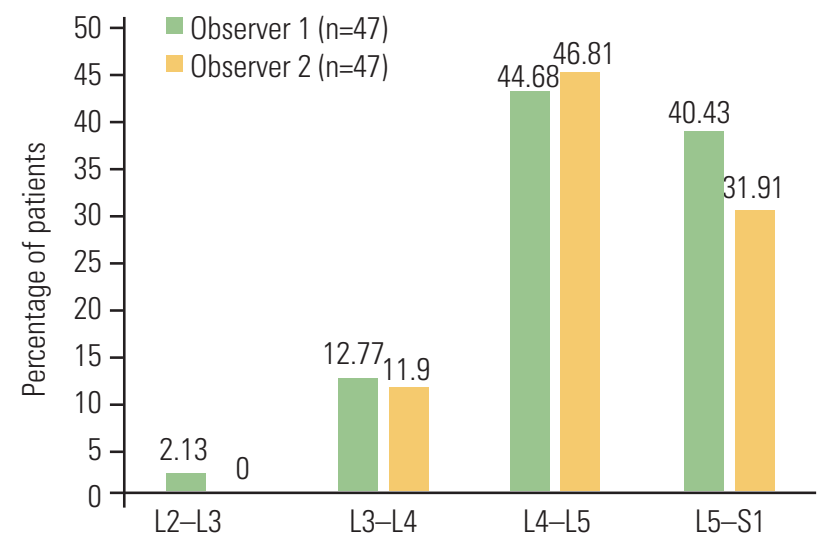

Fig. 2. Distribution of endplate cavities according to the levels of PEEK cages (\%). PEEK, polyetheretherketone.

months (range, 6-57 months). In 41 cases (63.08\%), the follow-up was $\geq 12$ months. The patients' demographic data are summarized in Table 1.

\section{Assessment of fusion}

Definite fusion was seen in 56 levels $(65.12 \%)$ by observer 1 versus 44 levels (51.16\%) by observer 2 (Fig. 1, Tables 2, $3)$. The strength of agreement was considered to be moderate $(\kappa=0.587)$. The $95 \%$ confidence interval $(95 \% \mathrm{CI})$ was $0.429-0.746$. 
Table 1. Baseline characteristics

\begin{tabular}{|c|c|}
\hline Characteristic & Value \\
\hline Age (yr) & $35.44 \pm 19.60$ (range, $26-67$ ) \\
\hline \multicolumn{2}{|l|}{ Gender (n=65) } \\
\hline Male & $21(32.31)$ \\
\hline Female & 44 (67.69) \\
\hline \multicolumn{2}{|c|}{ Diagnosis, according to level ( $\mathrm{n=86}$ ) } \\
\hline Prolapsed disc & $26(30.23)$ \\
\hline Stenosis & $6(6.98)$ \\
\hline Listhesis & 49 (56.97) \\
\hline Revision & $5(5.81)$ \\
\hline \multicolumn{2}{|l|}{ Fixation level $(n=86)$} \\
\hline L2-L4 & $2(2.33)$ \\
\hline L3-L4 & $2(2.33)$ \\
\hline L3-L5 & $16(18.60)$ \\
\hline L3-S1 & $11(12.79)$ \\
\hline L4-L5 & $14(16.28)$ \\
\hline L4-S1 & $20(23.26)$ \\
\hline L5-S1 & $21(24.42)$ \\
\hline \multicolumn{2}{|l|}{ No. of levels fixed $(n=86)$} \\
\hline 1 & $36(41.86)$ \\
\hline 2 & $38(44.18)$ \\
\hline 3 & $12(13.95)$ \\
\hline \multicolumn{2}{|c|}{ Level of polyetheretherketone insertion ( $n=86$ ) } \\
\hline $\mathrm{L} 2-\mathrm{L} 3$ & $1(1.16)$ \\
\hline L3- L4 & $11(12.79)$ \\
\hline$\llcorner 4-\mathrm{L} 5$ & $38(44.19)$ \\
\hline L5-S1 & $36(41.86)$ \\
\hline \multicolumn{2}{|l|}{ Follow-up (n=86) } \\
\hline$<12 \mathrm{mo}$ & $40(46.51)$ \\
\hline$\geq 12 \mathrm{mo}$ & $46(53.49)$ \\
\hline Follow-up duration (mo) & $16.5 \pm 10.1$ (range, 6-57) \\
\hline
\end{tabular}

Values are presented as mean \pm standard deviation (range) or number (\%).

\section{Endplate cavities}

The presence of endplate cavities was observed in 42 levels (48.84\%) by observer 1 versus 47 levels (54.65\%) by observer 2 (Figs. 1-3, Tables 2, 3). The strength of agreement was considered to be fair $(\kappa=0.373)$. The $95 \%$ CI was $0.179-0.568$.

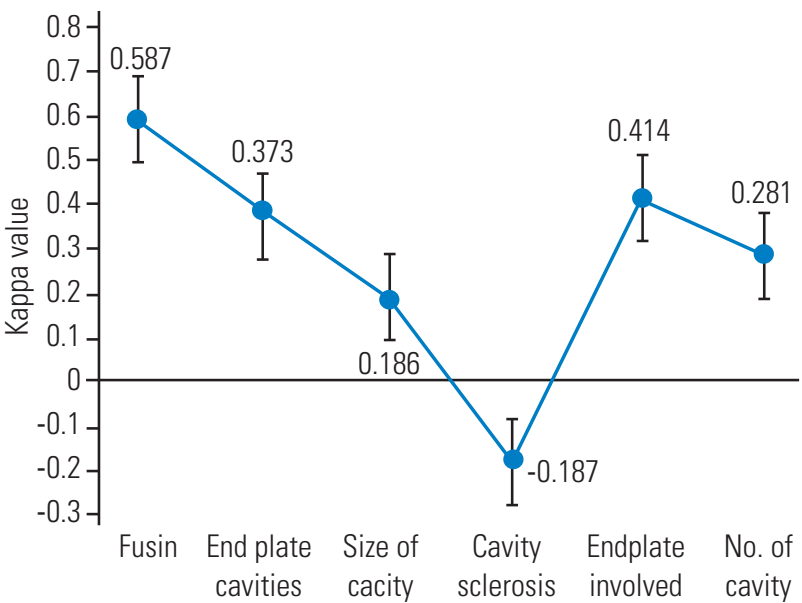

Fig. 3. Summary of the reliability of the fusion and endplate cavity characteristics.

\section{Location of endplate}

Endplates were most frequently located both caudally and cephalically in $36.04 \%$ of the levels by observer 1 . However, the cephalic location was most frequently identified (17\%) by observer 2 (Table 4 ). The strength of agreement was considered to be moderate $(\kappa=0.414)$, and the $95 \% \mathrm{CI}$ was $0.269-0.560$.

\section{Cavity size}

A significant variation in cavity sizes was reported between the observers (Table 5). The strength of agreement was considered to be poor $(\kappa=0.186)$, and the $95 \%$ CI was $0.088-0.284$.

\section{Single versus multiple cavities}

Most of the cavities were multiple. Single cavities were observed in 16 out of 86 levels (18.60\%) by observer 1 versus 29 levels (33.72\%) by observer 2 . The strength of agreement was considered to be fair $(\kappa=0.281)$, and the $95 \% \mathrm{CI}$ was $0.142-0.421$.

\section{Endplate sclerosis}

Significant variations in endplate sclerosis were also seen between the observers. The strength of agreement was worse than that expected by chance alone. The $\kappa$ was -0.187 , and the $95 \%$ CI was -0.305 to -0.069 . 
Table 2. Fusion status at the end of the follow-up

\begin{tabular}{lcccc} 
& \multicolumn{4}{c}{ Observer 2} \\
\cline { 2 - 5 } Observer 1 & Definite fusion & No fusion & Questionable fusion & Total \\
Definite fusion & $44(51.16)$ & $11(12.79)$ & $1(1.16)$ & $56(65.12)$ \\
No fusion & $1(1.16)$ & $18(20.93)$ & 0 & $19(22.09)$ \\
\hline Questionable fusion & $5(5.81)$ & $1(1.16)$ & $5(5.81)$ & $11(12.79)$ \\
\hline Total & $50(58.14)$ & $30(34.88)$ & $6(6.98)$ & $86(100.00)$ \\
\hline
\end{tabular}

Values are presented as frequency (\%).

Table 3. Fusion rate and presence of cavities according to the follow-up

\begin{tabular}{llcr} 
Duration (mo) & Criteria & Observer 1 & Observer 2 \\
\hline$<12$ & Definite fusion & $26 / 40(65.00)$ & $21 / 40(52.50)$ \\
\hline $12-24$ & Cavity present & $21 / 40(52.50)$ & $21 / 40(52.50)$ \\
\hline$>24$ & Definite fusion & $15 / 28(53.57)$ & $16 / 28(57.14)$ \\
\hline & Cavity present & $22 / 28(78.57)$ & $14 / 28(50.00)$ \\
\hline
\end{tabular}

Values are presented as frequency (\%).

Table 4. Location of endplate cavities

\begin{tabular}{lccccc} 
& \multicolumn{5}{c}{ Observer 2} \\
\cline { 2 - 6 } Observer 1 & Both & Caudal & No cavity & Cephalic & Total \\
Both & $13(15.11)$ & $3(3.49)$ & $12(13.95)$ & $3(3.49)$ & $31(36.04)$ \\
Caudal & 0 & $3(3.49)$ & 0 & 0 & $3(3.49)$ \\
No cavity & 0 & $5(5.81)$ & $28(32.56)$ & $6(6.98)$ & $39(45.35)$ \\
Cephalic & $1(1.16)$ & 0 & $4(4.65)$ & $8(9.30)$ & $13(15.12)$ \\
Total & $14(16.28)$ & $11(12.79)$ & $44(51.16)$ & $17(19.77)$ & $86(100.00)$ \\
\hline
\end{tabular}

Values are presented as frequency (\%).

Table 5. Endplate cavity size

\begin{tabular}{lccccc} 
& \multicolumn{5}{c}{ Observer 2} \\
Observer 1 & I & II & III & No cavity & Total \\
\cline { 2 - 6 } & $3(3.49)$ & $1(1.16)$ & $9(10.47)$ & $10(11.63)$ & $23(26.74)$ \\
II & $1(1.16)$ & 0 & $13(15.12)$ & $6(6.80)$ & $20(23.25)$ \\
NII & 0 & 0 & $4(4.65)$ & 9 & $4(4.65)$ \\
\hline Total & 0 & $2(2.33)$ & $9(10.47)$ & $28(32.39)$ & $39(45.35)$ \\
\hline
\end{tabular}

Values are presented as frequency (\%). 


\section{Factors affecting the presence of endplate cavities with various parameters}

The factors affecting the presence of endplate cavities were calculated using Pearson's correlation test. With both observers, neither the levels nor the follow-up period was significantly related to the presence of cavities. Significantly more unions than nonunions were detected by observer $1(p<0.0001)$. The differences detected by observer 2 were nearly significant $(p=0.05)$ (Table 6 ).

\section{Bullet-shaped versus kidney-shaped PEEK cages}

Tables 7 and 8 summarize the endplate cavity and fusion status of the two types of PEEK cages. There was no statistical difference between the cage type and cavities

Table 6. Association between the presence of endplate cavities with various parameters for observer 1 and observer 2

\begin{tabular}{lcc} 
Variable & $r$ & $p$-value \\
Observer 1 & & \\
$\quad$ Follow-up & -0.0296 & 0.7839 \\
\hline Fusion & -0.4288 & $<0.0001$ \\
\hline Level of PEEK insertion & -0.0508 & 0.6410 \\
\hline Observer 2 & & \\
\hline Follow-up & -0.0658 & 0.5460 \\
\hline Fusion & -0.2123 & 0.0500 \\
\hline Level of PEEK insertion & -0.0397 & 0.7146 \\
\hline
\end{tabular}

PEEK, polyetheretherketone.

* $p<0.05$. or fusion detected by both observers. Cage-related complications, including migration or retropulsion, were not recorded in our patients. No revision surgeries were performed.

\section{Discussion}

On the basis of our initial observations and recent articles, a high prevalence of unfavorable endplate changes after PEEK application in lumbar fusions surgery exists. We feel that the use of the term "cavity" is more appropriate because of the lack of any histological evidence for the other terms previously used to describe that phenomenon (osteolysis or cysts) [15-17]. Endplate cavities were seen in almost half of the levels in this study ( $48.84 \%$ by observer 1 versus $54.65 \%$ by observer 2). This figure was higher than previously reported in studies by Tanida et al. [16] (17.2\%) but slightly less than that reported by Nemoto et al. [15] (60\%). Despite the significant endplate changes observed in our study, only fair agreement existed between the observers $(\kappa=0.373)$. Moreover, our results revealed moderate reliability for endplate location $(\kappa=0.414)$, fair for cavity number $(\kappa=0.281)$, and poor for cavity size $(\kappa=0.186)$. We were unable to explain the reason for the differences. However, it should be noted that, on the basis of our results, the definition and characteristics of endplate changes following the use of PEEK should be reevaluated and standardized for better reliability.

Although the mechanism (s) responsible for the formation of vertebral endplate cavities remains unknown, several mechanisms based on biomechanical as well as

Table 7. Summary of the endplate cavity and fusion status with two types of polyetheretherketone cages (bullet shaped and kidney shaped) according to the level (total levels=86)

\begin{tabular}{|c|c|c|c|c|}
\hline \multirow{3}{*}{ Status } & \multicolumn{4}{|c|}{ Type of implant } \\
\hline & \multicolumn{2}{|c|}{ Bullet shaped cage } & \multicolumn{2}{|c|}{ Kidney shaped cage } \\
\hline & Observer 1 & Observer 2 & Observer 1 & Observer 2 \\
\hline \multicolumn{5}{|l|}{ No. of cavity } \\
\hline Yes & 34 & 30 & 13 & 12 \\
\hline No & 26 & 30 & 13 & 14 \\
\hline Total & 60 & 60 & 26 & 26 \\
\hline \multicolumn{5}{|l|}{ Fusion } \\
\hline Definite fusion & 38 & 34 & 17 & 16 \\
\hline Questionable and no fusion & 22 & 26 & 9 & 10 \\
\hline Total & 60 & 60 & 26 & 26 \\
\hline
\end{tabular}


Table 8. Association between the cage type (bullet shaped versus kidney shaped) and cavities and fusion for both observers

\begin{tabular}{lcc} 
& \multicolumn{2}{c}{$p$-value } \\
\cline { 2 - 3 } Variable & Observer 1 & Observer 2 \\
Cavities & 0.6241 & 0.6721 \\
Fusion & 0.7188 & 0.584 \\
\hline
\end{tabular}

biological PEEK incompatibility have been proposed. One of them is based on the lower stiffness of PEEK compared to titanium and the lack of sharp teeth on the cage, resulting in insufficient initial stability, which induces micromotions between the cage and the vertebral endplate. Micromotions may lead to microfractures, edema, focal bone resorption, and subsequent local necrotic regions, which may be similar to the formation of subchondral bone cysts in arthritic joints [16]. This explanation seems logical, and it indicated that cavities should be surrounded by sclerosis. Therefore, we evaluated sclerosis in our radiographs. However, our results showed less than poor reliability in the detection of sclerosis. Moreover, the endplate changes might have been vertebral osteolytic changes resulting from a reaction to the PEEK polymer, similar to that induced by cement in arthroplasties [15]. However, we have no pathological-based literature to support that hypothesis. Pre-existing cysts, seen as Modic changes similar to those in arthritic joints, could also be a possibility [16]. In addition, excessive endplate preparation might also be a factor, especially if the procedures were performed by less-experienced surgeons. Therefore, surgical skill is of the utmost importance in performing interbody fusions. Our surgeries were performed by two experienced consulting surgeons. We could postulate two other mechanisms that, to our knowledge, have never been described. The first might have occurred because of the habit of some surgeons to use the largest possible cage, which may cause injury to one or both of the endplates. The second mechanism could be subclinical low-grade infections. Our study excluded patients with frank postoperative infections. However, both mechanisms have to be considered in future studies.

The results of union/nonunion detections in this study should be interpreted with caution because of the relatively short follow-up and the moderate agreement between the assessments. The definite union rate was lower in patients with $<24$ months of follow-up and higher for those with $>24$ months follow-up $(77.7 \%$ and $72.2 \%$ for observer 1 and observer 2, respectively).

The previous high union rate $(100 \%)$ at 24 months reported by Brantigan et al. [18] was based only on the assessment of plain radiographs. Schimmel et al. [19] reported a high number of patients who underwent revision surgeries $(n=26,27.4 \%)$ mainly because of symptomatic pseudarthrosis for ALIF using PEEK cages. Nemoto et al. [15] reported a $96 \%$ union rate in the titanium group and $64 \%$ in the PEEK group at 12 months, which increased to $100 \%$ in the titanium group and $76 \%$ in the PEEK group at 24 months. They reported vertebral osteolysis in $60 \%$ of the cases with nonunion. By contrast, this abnormal finding was not present in the titanium group [15].

In addition, Tanida et al. [16] reported postoperative bone union rates of $75.2 \%$ and $74.5 \%$ at 1 year and $82.8 \%$ and $80.4 \%$ at 2 years for the titanium and PEEK groups, respectively. The rates of positive cyst signs in the titanium and PEEK groups were $17.2 \%$ and $13.7 \%$, respectively. The nonunion rate with positive cyst signs was $100 \%$ in both groups at 1 year [16]. Similarly, we found the percentage of cavities to be higher in patients with nonunions ( $p<0.0001$ for the first observer and $p=0.05$ for the second observer). We agree with Fujibayashi et al. [17], who reported that the clinical relevance of a vertebral endplate cyst might be an early predictor of nonunion.

Our finding of only moderate agreement in the detection of bony unions might reflect the high dependence and considerable variability between observers despite the criteria applied. In our opinion, it could also mean that the radiological evidence of healing after the use of PEEK cages was not clear or strong enough to reach a consensus. Further CT-based studies could support that hypothesis. Moreover, we do not support the proposed simple evaluation of radiologic fusion in PEEK cages by using only anteroposterior and lateral dynamic radiographs, and those evaluations should be interpreted with great caution.

A clear disadvantage of PEEK cages is their smooth and hydrophobic surface, which results in poorer primary stability. Furthermore, the resulting low surface energy negatively effects bone growth onto the implant. That is why many surgeons use expensive growth factors, such as rhBMP, to improve interbody fusion [20,21].

One strategy to improve primary stability and osteointegration is to modify the implant's surface by chemical or physical treatment or by adding an osteoconductive layer, usually hydroxyapatite or titanium, thereby increasing the 
contact area and roughness [22]. Recently, the first author of this study published the first results of a new vacuum plasma sprayed titanium-coated carbon/PEEK composite cage for lumbar interbody fusion. A significant degree of clinical improvement was achieved, in addition to radiological unions in all patients with a mean 24-month follow-up. However, the fusion was evaluated only by plain radiographs [23].

Our study had several limitations. First was the sample size, which needs to be expanded to provide more power and reliability. Second was the retrospective nature of the study. A prospective study could identify the evolution of cavities with respect to fusions. Such a study could help in answering whether the endplate cavities found in the initial follow-up period are predictive factors for future nonunions. Third, unfortunately, we did not include preoperative CTs to exclude pre-existing Modic or arthritic endplate changes because they were available. Fourth, we did not check the bone mineral density in all cases. Correlative studies between bone quality and cyst formation should be conducted in the future [17]. Fifth is that the follow-up period did not extend long enough to study the long-term effects, and therefore, longer follow-up studies are needed. Furthermore, we did not include patient characteristics that were likely to affect outcome and fusion, such as smoking and body mass index. Although this information is important when considering clinical outcomes, this was not the focus of the current study. Finally, two surgeons performed all surgeries in the current study. Although the procedures were consistent in principle, it was beyond the scope of this article to include any further clinical or postoperative implications or correlations.

\section{Conclusions}

There was a high rate of unfavorable radiographic findings in the form of endplate cavities in PLIF cases using PEEK cages. A more comprehensive classification for the assessment of fusions and endplate cavities should be formulated. We strongly recommend further CT-based studies with larger sample size and longer follow-up.

\section{Conflict of Interest}

No potential conflict of interest relevant to this article was reported.

\section{Author Contributions}

Tarek Elfiky: study design, radiographic assessment and writing the manuscript; Nirmal Dhananjay Patil: study design, radiographic assessment and statistical analysis; Yasser Allam: radiographic assessment and critical review; and Raafat Ragab: critical review.

\section{References}

1. Elgafy H, Olson D, Liu J, Lewis C, Semaan H. Effectiveness and safety of transforaminal lumbar interbody fusion in patients with previous laminectomy. Eur Spine J 2015;24:810-6.

2. Rousseau MA, Lazennec JY, Saillant G. Circumferential arthrodesis using PEEK cages at the lumbar spine. J Spinal Disord Tech 2007;20:278-81.

3. Rihn JA, Patel R, Makda J, et al. Complications associated with single-level transforaminal lumbar interbody fusion. Spine J 2009;9:623-9.

4. Pavlov PW, Meijers H, van Limbeek J, et al. Good outcome and restoration of lordosis after anterior lumbar interbody fusion with additional posterior fixation. Spine (Phila Pa 1976) 2004;29:1893-900.

5. Van Dijk M, Smit TH, Sugihara S, Burger EH, Wuisman PI. The effect of cage stiffness on the rate of lumbar interbody fusion: an in vivo model using poly(llactic acid) and titanium cages. Spine (Phila Pa 1976) 2002;27:682-8.

6. Spruit M, Falk RG, Beckmann L, Steffen T, Castelein RM. The in vitro stabilising effect of polyetheretherketone cages versus a titanium cage of similar design for anterior lumbar interbody fusion. Eur Spine J 2005;14:752-8.

7. Blumenthal SL, Gill K. Can lumbar spine radiographs accurately determine fusion in postoperative patients?: correlation of routine radiographs with a second surgical look at lumbar fusions. Spine (Phila Pa 1976) 1993;18:1186-9.

8. McAfee PC, Boden SD, Brantigan JW, et al. Symposium: a critical discrepancy: a criteria of successful arthrodesis following interbody spinal fusions. Spine (Phila Pa 1976) 2001;26:320-34.

9. Pavlov PW, Spruit M, Havinga M, Anderson PG, van Limbeek J, Jacobs WC. Anterior lumbar interbody fusion with threaded fusion cages and autologous 
bone grafts. Eur Spine J 2000;9:224-9.

10. Cho CB, Ryu KS, Park CK. Anterior lumbar interbody fusion with stand-alone interbody cage in treatment of lumbar intervertebral foraminal stenosis: comparative study of two different types of cages. J Korean Neurosurg Soc 2010;47:352-7.

11. Smit TH, Muller R, van Dijk M, Wuisman PI. Changes in bone architecture during spinal fusion: three years follow-up and the role of cage stiffness. Spine (Phila Pa 1976) 2003;28:1802-9.

12. Ferguson SJ, Visser JM, Polikeit A. The long-term mechanical integrity of non-reinforced PEEK-OPTIMA polymer for demanding spinal applications: experimental and finite-element analysis. Eur Spine J 2006;15:149-56.

13. Vadapalli S, Sairyo K, Goel VK, et al. Biomechanical rationale for using polyetheretherketone (PEEK) spacers for lumbar interbody fusion: a finite element study. Spine (Phila Pa 1976) 2006;31:E992-8.

14. Cabraja M, Oezdemir S, Koeppen D, Kroppenstedt S. Anterior cervical discectomy and fusion: comparison of titanium and polyetheretherketone cages. BMC Musculoskelet Disord 2012;13:172.

15. Nemoto O, Asazuma T, Yato Y, Imabayashi H, Yasuoka H, Fujikawa A. Comparison of fusion rates following transforaminal lumbar interbody fusion using polyetheretherketone cages or titanium cages with transpedicular instrumentation. Eur Spine J 2014;23:2150-5.

16. Tanida S, Fujibayashi S, Otsuki B, et al. Vertebral endplate cyst as a predictor of nonunion after lumbar interbody fusion: comparison of titanium and polyetheretherketone cages. Spine (Phila Pa 1976) 2016;41:E1216-22.
17. Fujibayashi S, Takemoto M, Izeki M, Takahashi Y, Nakayama T, Neo M. Does the formation of vertebral endplate cysts predict nonunion after lumbar interbody fusion? Spine (Phila Pa 1976) 2012;37:E1197202.

18. Brantigan JW, Steffee AD, Lewis ML, Quinn LM, Persenaire JM. Lumbar interbody fusion using the Brantigan I/F cage for posterior lumbar interbody fusion and the variable pedicle screw placement system: two-year results from a Food and Drug Administration investigational device exemption clinical trial. Spine (Phila Pa 1976) 2000;25:1437-46.

19. Schimmel JJ, Poeschmann MS, Horsting PP, Schonfeld DH, van Limbeek J, Pavlov PW. PEEK cages in lumbar fusion: mid-term clinical outcome and radiologic fusion. Clin Spine Surg 2016;29:E252-8.

20. Han CM, Lee EJ, Kim HE, et al. The electron beam deposition of titanium on polyetheretherketone (PEEK) and the resulting enhanced biological properties. Biomaterials 2010;31:3465-70.

21. Devine DM, Hahn J, Richards RG, Gruner H, Wieling R, Pearce SG. Coating of carbon fiber-reinforced polyetheretherketone implants with titanium to improve bone apposition. J Biomed Mater Res B Appl Biomater 2013;101:591-8.

22. Takemoto M, Fujibayashi S, Neo M, et al. A porous bioactive titanium implant for spinal interbody fusion: an experimental study using a canine model. J Neurosurg Spine 2007;7:435-43.

23. Hoppe S, Albers CE, Elfiky T, et al. First results of a new vacuum plasma sprayed (VPS) titanium-coated carbon/PEEK composite cage for lumbar interbody fusion. J Funct Biomater 2018;9:E23. 